\title{
Influence of reconstruction kernels on the accuracy of CT-derived fractional flow reserve
}

\author{
Fabian Ammon ${ }^{1}$ (D) Maximilian Moshage ${ }^{1} \cdot$ Silvia Smolka $^{1} \cdot$ Markus Goeller $^{1} \cdot$ Daniel O. Bittner $^{1}$. \\ Stephan Achenbach ${ }^{1} \cdot$ Mohamed Marwan ${ }^{1}$
}

Received: 1 July 2021 / Revised: 31 August 2021 / Accepted: 17 September 2021 / Published online: 4 November 2021

(C) The Author(s) 2021

\begin{abstract}
Objectives We evaluated the influence of image reconstruction kernels on the diagnostic accuracy of CT-derived fractional flow reserve $\left(\mathrm{FFR}_{\mathrm{CT}}\right)$ compared to invasive FFR in patients with coronary artery disease.

Methods Sixty-nine patients, in whom coronary CT angiography was performed and who were further referred for invasive coronary angiography with FFR measurement via pressure wire, were retrospectively included. CT data sets were acquired using a third-generation dual-source CT system and rendered with medium smooth (Bv40) and sharp (Bv49) reconstruction kernels. $\mathrm{FFR}_{\mathrm{CT}}$ was calculated on-site using prototype software. Coronary stenoses with invasive $\mathrm{FFR} \leq 0.80$ were classified as significant. Agreement between FFR $_{\mathrm{CT}}$ and invasive FFR was determined for both reconstruction kernels.

Results One hundred analyzed vessels in 69 patients were included. Twenty-five vessels were significantly stenosed according to invasive FFR. Using a sharp reconstruction kernel for $\mathrm{FFR}_{\mathrm{CT}}$ resulted in a significantly higher correlation with invasive FFR $(r=0.74, p<0.01$ vs. $r=0.58, p<0.01 ; p=0.04)$ and a higher AUC in ROC curve analysis to correctly identify/ exclude significant stenosis ( $\mathrm{AUC}=0.92$ vs. $\mathrm{AUC}=0.82$ for sharp vs. medium smooth kernel, respectively, $p=0.02$ ). A $\mathrm{FFR}_{\mathrm{CT}}$ value of $\leq 0.8$ using a sharp reconstruction kernel showed a sensitivity of $88 \%$ and a specificity of $92 \%$ for detecting ischemia-causing lesions, resulting in a diagnostic accuracy of $91 \%$. The medium smooth reconstruction kernel performed worse (sensitivity $60 \%$, specificity $89 \%$, accuracy $82 \%$ ).

Conclusion Compared to invasively measured FFR, $\mathrm{FFR}_{\mathrm{CT}}$ using a sharp image reconstruction kernel shows higher diagnostic accuracy for detecting lesions causing ischemia, potentially altering decision-making in a clinical setting.

Key Points

- Image reconstruction parameters influence the diagnostic accuracy of simulated fractional flow reserve derived from coronary computed tomography angiography.

- Using a sharp kernel image reconstruction algorithm delivers higher diagnostic accuracy compared to medium smooth kernel image reconstruction (gold standard invasive fractional flow reserve).
\end{abstract}

Keywords Computed tomography angiography · Image processing, computer-assisted · Fractional flow reserve, myocardial $\cdot$ Coronary artery disease

\section{Abbreviations}

AUC Area under the ROC curve

bpm Beats per minute

CAD Coronary artery disease

cCTA Coronary computed tomography angiography

CT Computed tomography

Fabian Ammon

fabian.ammon@uk-erlangen.de

Department of Cardiology, Friedrich-Alexander University Erlangen-Nürnberg (FAU), Ulmenweg 18, 91054 Erlangen, Germany

$\begin{array}{ll}\text { CX } & \text { Circumflex coronary artery } \\ \text { FFR } & \text { Fractional flow reserve } \\ \text { FFR }_{\text {CT }} & \text { CT-derived FFR } \\ \text { i.v. } & \text { Intravenous } \\ \text { IQR } & \text { Interquartile range } \\ \text { LAD } & \text { Left anterior descending artery } \\ \text { p.o. } & \text { Per os } \\ \text { RCA } & \text { Right coronary artery } \\ \text { ROC } & \text { Receiver operating characteristic }\end{array}$




\section{Introduction}

Coronary computed tomography (CT) is an established anatomic imaging modality for exclusion and detection of coronary artery stenoses; it carries a "class I" indication for the workup of coronary artery disease (CAD) in specific patient populations. However, assessment of the physiologic relevance of coronary stenoses based on anatomy remains difficult and ischemia testing for guiding revascularization decisions is recommended.

In the absence of sufficient information from previous stress testing, the hemodynamic significance of coronary stenoses can be assessed with invasive fractional flow reserve (FFR). An FFR-based approach has repeatedly been shown to be superior to relying on angiography alone regarding clinical outcome [1-4], and FFR-based decision-making has emerged as the gold standard for performing or deferring percutaneous coronary intervention [5].

With the help of computational fluid dynamics, noninvasive FFR values are generated from coronary computed tomography angiography (cCTA) data sets (CT-derived FFR, $\mathrm{FFR}_{\mathrm{CT}}$ ) and thus add hemodynamic information to pure anatomic images [6].

Several trials have reported a good correlation of $\mathrm{FFR}_{\mathrm{CT}}$ with invasive FFR. Furthermore, compared to invasive FFR, the diagnostic accuracy of $\mathrm{FFR}_{\mathrm{CT}}$ for detecting ischemia-causing coronary lesions could be shown to be better than cCTA alone [7-9]. Minimizing artifacts and the use of nitroglycerin and beta blocker are key factors for achieving a high diagnostic accuracy of $\mathrm{FFR}_{\mathrm{CT}}$ [7-9].

Since the determination of $\mathrm{FFR}_{\mathrm{CT}}$ requires a high degree of anatomic information and spatial resolution, it is conceivable that the choice of reconstruction kernels used in the process of generating CT image data sets impacts the results of $\mathrm{FFR}_{\mathrm{CT}}$. Reconstruction kernels can be compared to image filters that determine the balance between image noise and spatial resolution within the obtained data set. A "sharp" reconstruction kernel is very useful for assessment of coronary arteries when pronounced atherosclerosis is present and especially in the presence of calcifications. The influence of image reconstruction kernels on the diagnostic performance of $\mathrm{FFR}_{\mathrm{CT}}$ has not yet been investigated.

The aim of this study was to assess the influence of using "sharp" versus "smooth" reconstruction kernels on the accuracy of $\mathrm{FFR}_{\mathrm{CT}}$, which was determined by comparison to invasively measured FFR.

\section{Materials and methods}

\section{Study design and patient population}

This is a single-center, retrospective analysis. Patients referred for cCTA between April 2015 and February 2019 were screened for inclusion in this analysis. Exclusion criteria were non-diagnostic cCTA data sets, coronary anomalies, the presence of a chronic total obstruction or prior stent implantation in the vessel of interest, and any status post coronary artery bypass grafting as well as the presence of ostial left main or ostial right coronary stenosis. Sixty-nine patients with an invasive coronary angiogram within 2 months after cCTA and invasive FFR measurement during which the wire position was unambiguously documented by fluoroscopy were included in this study.

\section{CT data acquisition and image reconstruction}

Coronary CT angiography was performed using a third-generation dual-source CT system (Somatom Force, Siemens Healthineers). Patients with heart rates $>60 \mathrm{bpm}$ were given atenolol p.o. and/or metoprolol i.v. All patients received nitroglycerin sublingually prior to the scan. The acquisition protocol was chosen according to patient characteristics. ECG-triggered prospective axial acquisition was performed in most patients. Patients with persistent heart rates $>70 \mathrm{bpm}$ in spite of medication or with frequent ectopic beats were examined using spiral acquisition with retrospectively ECGgated image reconstruction. Patients $<45$ years old with heart rates $<55 \mathrm{bpm}$ were examined using prospectively ECG-triggered high-pitch spiral acquisition. Coronary calcium screening CT was performed in most patients before administering contrast agent. The scan range extended from the proximal ascending aorta to the caudal aspect of the heart. For coronary CT angiography, tube voltage (ranging from 90 to $120 \mathrm{mV}$ ) and tube current time product (450 to $650 \mathrm{mAs}$ ) were adjusted to the patient's body weight and calcium score. Contrast agent transit time was determined by giving a bolus of $10 \mathrm{ml}$ of contrast agent (Ultravist $370 \AA$, Bayer vital GmbH GB Pharma). For CT angiography, $50 \mathrm{ml}$ of contrast agent at a flow rate of $5 \mathrm{ml} / \mathrm{s}$, followed by a $50-\mathrm{ml}$ saline chaser ( $20 \%$ contrast agent) at the same flow rate, was injected.

All image data sets were reconstructed with a slice thickness of $0.5 \mathrm{~mm}$, increment of $0.3 \mathrm{~mm}$, and a matrix of 512 . Iterative reconstruction (Admire ${ }^{\circledR}$, Siemens Healthineers) at a strength level of 2 was used for all data sets. Reconstructions were rendered using both a medium soft convolution kernel (Siemens Bv40) and a sharp convolution kernel (Siemens Bv49), with otherwise unchanged parameters (Fig. 1).

\section{Calculation of $F F R_{C T}$}

FFR $_{\mathrm{CT}}$ was calculated on-site using a PC-based prototype software (cFFR version 3.0, Siemens Healthineers). The program automatically renders the centerline and lumen of the vessels. Manual correction by a user blinded to 

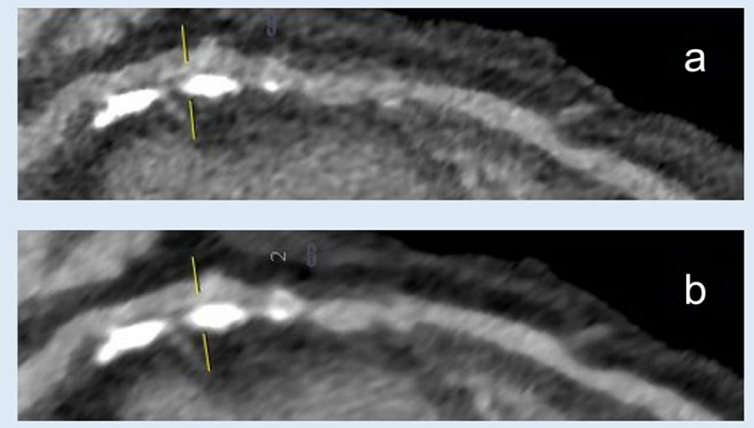

Fig. 1 cCTA image with a longitudinal section of the left anterior descending artery with a (a) sharp Bv49 kernel and (b) medium smooth Bv40 kernel

invasive FFR was done where necessary. When completed, a 3D model of the coronary tree is generated with FFR values at any point on the vessel. After identifying the exact location of the FFR wire in the invasive coronary angiogram, the $\mathrm{FFR}_{\mathrm{CT}}$ value at the identical location of the simulated coronary tree was noted and compared to the invasive FFR value (Figs. 2 and 3).

\section{Invasive FFR measurement}

Invasive FFR was measured using PressureWire X Guidewire (St. Jude Medical/Abbott) after producing hyperemia with adenosine intracoronary. Coronary stenoses with invasively measured FFR $\leq 0.80$ were classified as hemodynamically significant. Agreement between $\mathrm{FFR}_{\mathrm{CT}}$ using both reconstruction parameters was compared to invasive FFR.

\section{Statistical analyses}

All statistical analyses were done with IBM $®$ SPSS $®$ Statistics (version 24). Continuous variables are presented as mean \pm standard deviation or median (interquartile range [IQR]). Wilcoxon test was used to compare groups. Results
Fig. 2 a Invasive angiogram with an FFR wire placed in the distal third of the right coronary artery. b 3D CT reconstruction of the coronary tree showing FFR $_{\text {CT }}$

Fig. 3 a Invasive angiogram with an FFR wire placed in the distal left anterior descending artery. b 3D CT reconstruction of the coronary tree showing $\mathrm{FFR}_{\mathrm{CT}}$
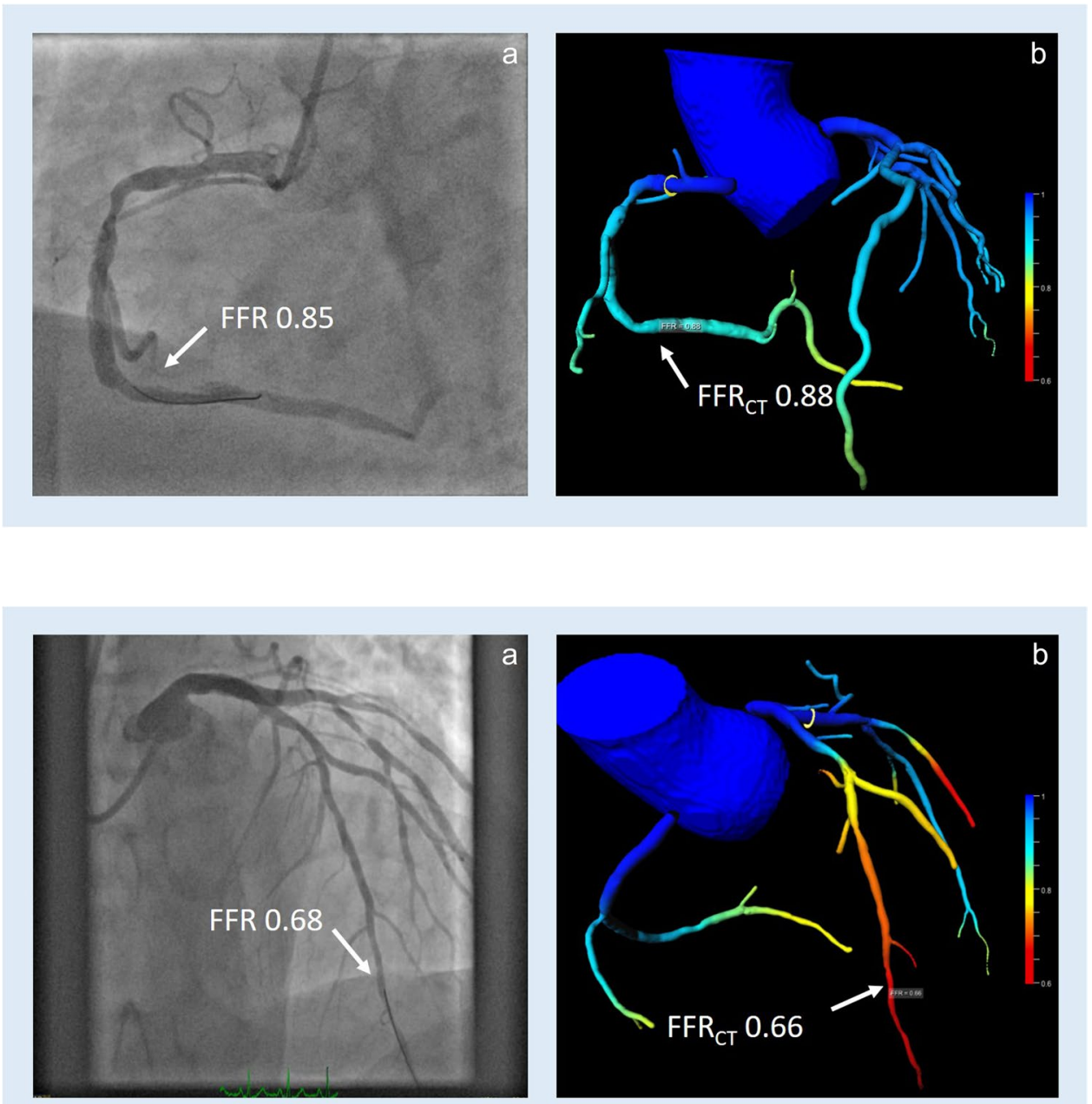
were deemed significant with a $p$ value $<0.05$. Receiver operating characteristic (ROC) curve analyses were performed and ROC curves and correlations compared with MedCalc Software Ltd.

\section{Results}

Out of 72 patients screened for inclusion in this analysis, 3 patients had to be excluded because of insufficient image quality of cCTA for the calculation of $\mathrm{FFR}_{\mathrm{CT}}$ and one patient due to technical reasons with $\mathrm{FFR}_{\mathrm{CT}}$ measurement. Sixtynine patients (mean age $63 \pm 10$ years, 18 women) were included in this study. Baseline clinical characteristics are shown in Table 1.

Invasive coronary angiography was performed after a mean time of 8 days after cCTA. A total of 100 vessels were investigated both by invasive and CT-based FFR. The invasive angiographic lumen reduction of the target vessel ranged between 30 and $90 \%$ on visual analysis (mean 57\%). Twenty-seven vessels had a degree of stenosis of $30-<50 \%$, 44 vessels showed a stenosis of $50-<70 \%$, while in 29 vessels a lumen reduction of $70-90 \%$ was found. In case of multiple stenoses, the pressure wire was placed after the most distal stenosis. The median invasive FFR value was 0.87 (IQR 0.14 ), and a total of 25 analyzed vessels ( $25 \%$ of all vessels) were classified as hemodynamically significantly stenosed due to an invasive FFR value of $\leq 0.8$.

In most cases (57/100), the left anterior descending artery (LAD) with its side branches was the vessel of interest, followed by the circumflex coronary artery (CX, 27/100) and the right coronary artery (RCA, 16/100). The hemodynamic relevance of lesions according to invasive FFR was detected in the LAD territory in 20 cases (35\% of all measured LAD),

Table 1 Demographic and clinical characteristics of included patients

\begin{tabular}{ll}
\hline & All patients $(N=69)$ \\
\hline $\begin{array}{l}\text { Mean age—yr } \\
\text { Known coronary artery disease* (CAD)—no. }\end{array}$ & $63 \pm 10$ \\
$\quad(\%)$ & $55(80)$ \\
Arterial hypertension—no. (\%) & $11(16)$ \\
Diabetes mellitus—no. (\%) & $49(71)$ \\
Hyperlipoproteinemia—no. (\%) & $21(30)$ \\
Positive family history for CAD—no. (\%) & $28(41)$ \\
Prior or current smoker—no. $(\%)$ & $27.3 \pm 3.9$ \\
Body mass index-kg/m ${ }^{2}$ & $56.6 \pm 7.0$ \\
Mean echocardiographic ejection fraction—\% & $57.2 \pm 15.8$ \\
Angiographic degree of stenosis—\% & $33(48)$ \\
Pure diagnostic coronary angiography—no. & \\
$\quad(\%)$ &
\end{tabular}

*Previous percutaneous coronary intervention not in the analyzed vessel in the CX territory in 3 cases ( $11 \%$ of all measured CX), and in the RCA in 2 cases (13\% of all measured RCA). There was a significant association between the vessel of interest and the severity of the stenosis $(p=0.027)$.

The median $\mathrm{FFR}_{\mathrm{CT}}$ using a medium smooth reconstruction kernel (Bv40) was 0.87 (IQR 0.11), and the median FFR $_{\mathrm{CT}}$ using a sharp reconstruction kernel (Bv49) was 0.87 (IRQ $0.13)$. While $F_{F R}$ values simulated with $B v 40$ were not relevantly different compared with invasive FFR values $(p=0.26)$, this was the case when simulated with $\operatorname{Bv} 49(p=0.017)$.

Using the sharp reconstruction kernel, a significantly closer correlation of $\mathrm{FFR}_{\mathrm{CT}}$ with invasive FFR was observed in comparison to medium smooth reconstruction kernel ( $r=0.74, p<0.0001$ vs $r=0.58, p<0.0001$; direct comparison of correlation coefficients $p=0.04$; scatterplots Figs. 4 and 5). ROC curve analysis showed an area under the ROC curve (AUC) of $0.92(p<0.001)$ for $\mathrm{FFR}_{\mathrm{CT}}$ using a sharp reconstruction kernel to correctly identify or exclude hemodynamically significant stenosis compared to invasive FFR, whereas a smaller AUC of $0.82(p<0.001)$ was observed for a medium smooth reconstruction kernel. This difference of AUC between $\mathrm{Bv} 49$ and $\mathrm{Bv} 40 \mathrm{FFR}_{\mathrm{CT}}$ was statistically significant $(p=0.02)$. A $\mathrm{FFR}_{\mathrm{CT}}$ value of $\leq 0.8$ using sharp reconstruction kernel data sets showed a sensitivity of $88 \%$ and a specificity of $92 \%$ to detect diseased vessels causing ischemia compared to the invasive gold standard, resulting in an accuracy of $91 \%$. The medium smooth kernel data sets performed significantly worse, having a sensitivity of $60 \%$ and specificity of $89 \%$, resulting in

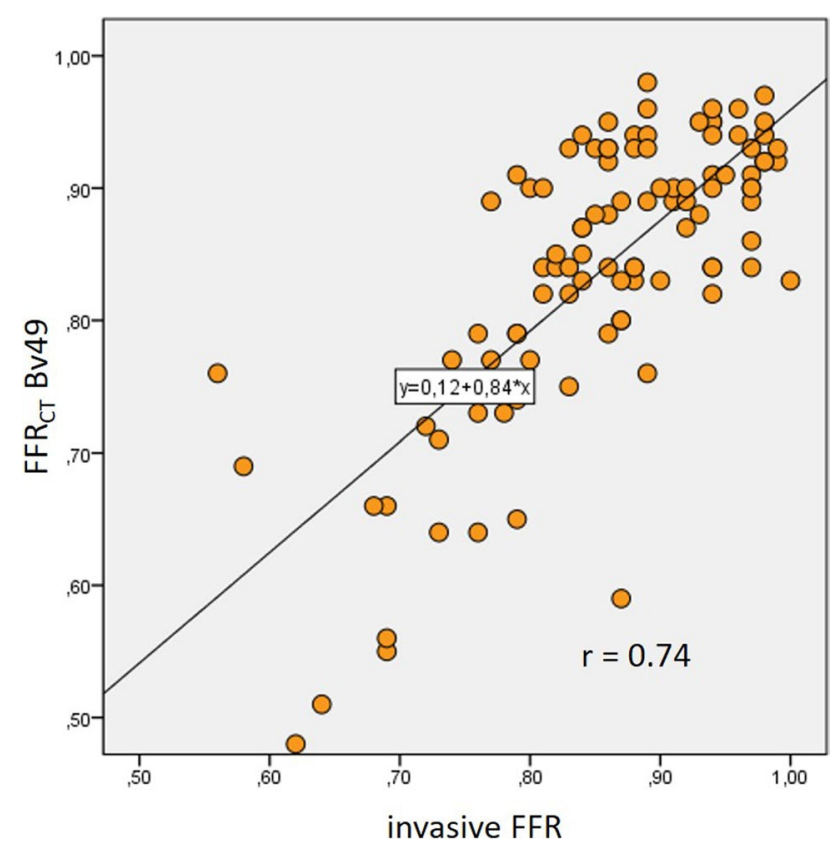

Fig. 4 Scatterplot with $\mathrm{FFR}_{\mathrm{CT}}$ Bv49 and invasive FFR 


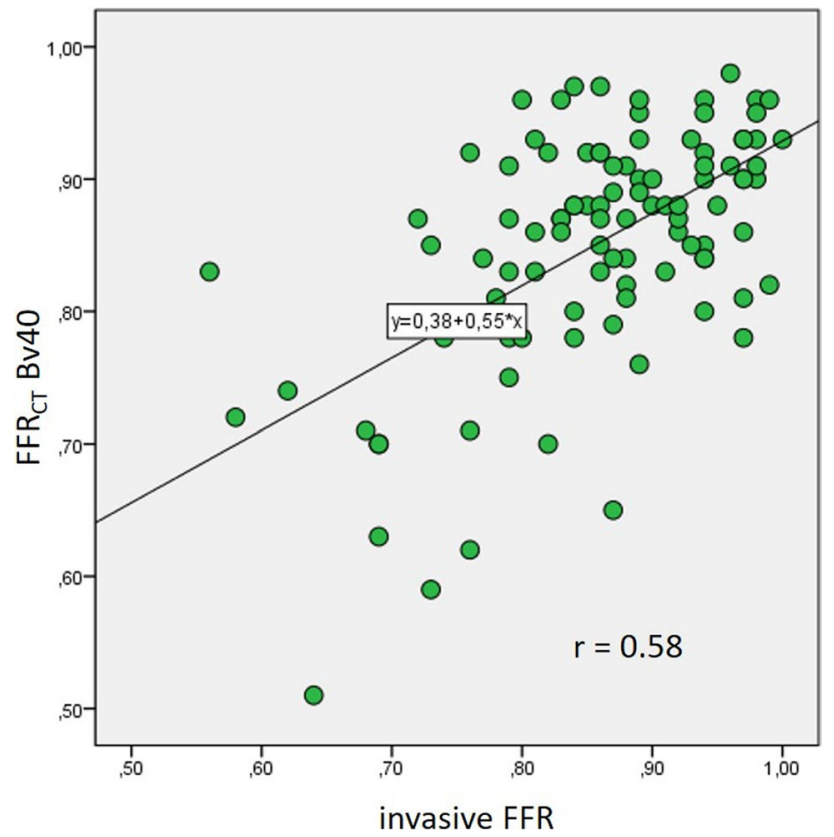

Fig. 5 Scatterplot with $\mathrm{FFR}_{\mathrm{CT}}$ Bv40 and invasive FFR

an accuracy of $82 \%$ when using the same $\mathrm{FFR}_{\mathrm{CT}}$ cut-off value of $\leq 0.8$. Statistical analysis [10] demonstrated the superiority of sharp kernel reconstructions regarding diagnostic accuracy when compared to medium smooth kernel reconstructions $(p=0.02)$.

In 46 patients, only one vessel was assessed for FFR whereas in 23 patients, more than one vessel was assessed. On a per-patient basis with one pathologic invasive FFR value indicating the presence of relevant stenoses, $\mathrm{FFR}_{\mathrm{CT}}$ with a Bv49 reconstruction kernel was able to correctly classify 61 patients. In 5 patients, the severity of stenosis was overestimated, and in 3 patients, relevant stenoses were not detected (sensitivity $88 \%$, specificity $89 \%$, diagnostic accuracy $88 \%$ ). Using a Bv40 reconstruction kernel, only 57 patients could be correctly classified with 5 patients overestimating and 7 patients underestimating the degree of stenosis severity (sensitivity $71 \%$, specificity $89 \%$, diagnostic accuracy $83 \%$ ). The numerically higher diagnostic accuracy of a sharp reconstruction kernel was not significantly superior to a medium smooth reconstruction kernel on a per-patient basis $(p=0.14)$.

\section{Discussion}

To the best of our knowledge, this is the first study to evaluate the influence of the image reconstruction kernel on $\mathrm{FFR}_{\mathrm{CT}}$ values. We could show that $\mathrm{FFR}_{\mathrm{CT}}$ using a sharper reconstruction kernel had a significantly better correlation with invasive FFR compared to a medium smooth reconstruction kernel. These results are of clinical relevance as patient-related decision-making concerning further diagnostic workup or invasive assessment critically depends on the accuracy of the $\mathrm{FFR}_{\mathrm{CT}}$ values. The higher spatial resolution of CT data sets generated by a sharper reconstruction kernel outweighs the disadvantage of increased noise, and therefore, the preferred kernel should render high-spatial-resolution data sets (often termed "sharp"). High image quality and high spatial resolution are key factors for obtaining accurate $\mathrm{FFR}_{\mathrm{CT}}$ results since the hemodynamic significance of a lesion is partly affected by the depicted degree of stenosis length and vessel diameter. Presumably, the enhanced spatial resolution offered by sharper reconstruction kernels improves automatic delineation of coronary centerlines with better lumen depiction and subsequent manual corrections can be done more confidently when needed.

Baumann and Renker et al compared invasive FFR with $\mathrm{FFR}_{\mathrm{CT}}$ by a prior version of the software used in our study. They showed a correlation similar to ours between $\mathrm{FFR}_{\mathrm{CT}}$ and invasively measured FFR $(r=0.74, p<0.0001)$ in a cohort of 28 patients/36 vessels [11]. In another trial with 53 patients and 67 lesions, the results with on-site $\mathrm{FFR}_{\mathrm{CT}}$ calculation were similar: a reasonable correlation of $\mathrm{FFR}_{\mathrm{CT}}$ with invasive FFR $(r=0.66, p<0.001)$ and increased diagnostic performance of $\mathrm{FFR}_{\mathrm{CT}}$ over cCTA alone for the detection of hemodynamically relevant stenosis defined by an invasive FFR $<0.8$ [12]. The image reconstruction kernel was not specified in the first trial, but in the second publication, image reconstruction was performed using a vascular kernel, B26f, which corresponds to a medium smooth reconstruction kernel. A study with 71 patients/91 vessels performed in our department could demonstrate a higher correlation $(r=0.85)$ and high diagnostic accuracy for $\mathrm{FFR}_{\mathrm{CT}}$ compared to invasive FFR by using the sharp Bv49 kernel [13].

The mean diameter reduction in the target lesion was $57 \%$ in our study. Invasive FFR is especially suitable to evaluate lesions with intermediate stenosis, as stenosis as low as $30-50 \%$ can already have hemodynamic relevance [1] [14], while no additional benefit has been shown for lesions showing $>90 \%$ stenosis [15]. Thus, our study investigated the effect of image reconstruction kernels in a study population, which represents the daily life challenge of evaluating the usefulness of performing a percutaneous coronary intervention.

Coronary CT angiography is recommended by the guidelines of the European Society of Cardiology to clarify whether CAD causes chest pain when clinical assessment alone cannot exclude CAD [16]. However, the low specificity of cCTA to rule out hemodynamic CAD sets patients at risk of unnecessary non-invasive testing or invasive coronary 
angiography $[17,18]$. The initial SCOT-HEART trial roused suspicion of increased invasive testing of patients presenting with chest pain who received coronary CT angiography as a first-line diagnostic tool for evaluation of chest pain [19]. However, the 5-year results of the SCOT-HEART trial could not only show a decrease in the primary endpoint, but also that the higher rate of invasive coronary angiography could only be observed in the first few months, while later no difference could be found [20]. Potentially using non-invasive FFR measurement for evaluating coronary stenoses in coronary CT angiography could have helped to reduce the number of early coronary angiographies while maintaining the positive long-term effect of increased use of antithrombotic and cholesterol-lowering agents in this cohort. Curzen et al could show that the availability of $\mathrm{FFR}_{\mathrm{CT}}$ values changes the treatment plan in about one-third of patients when added to standard coronary CT angiography [21]. The PLATFORM study also demonstrated that off-site $\mathrm{FFR}_{\mathrm{CT}}$ can safely reduce the number of invasive coronary angiographies without prognostic disadvantages in the group of patients with canceled invasive diagnostic in 1 year follow-up [22].

We could show that $\mathrm{FFR}_{\mathrm{CT}}$ performed with data reconstructed with a sharp kernel shows better results when compared to invasive FFR than a medium smooth kernel. The use of a sharp reconstruction kernel thus can help to achieve better diagnostic accuracy of non-invasive $\mathrm{FFR}_{\mathrm{CT}}$ algorithms.

Several limitations need to be acknowledged in this study. The number of patients and FFR-measured vessels are relatively small. Particularly, the number of pathologic invasive FFR values was low with 25 vessels. However, this is no sign of the low specificity of cCTA, as severely stenosed vessels were frequently not FFR-measured but directly intervened and only the intermediate stenoses were evaluated by FFR. With a mean angiographic degree of stenosis of $57 \%$, it was to be expected that the number of pathologic FFR is rather low, owing that even in vessels with 50 to $70 \%$ stenosis, hemodynamic significance of the lesion is present only in about one-third of cases [15]. The predominance of left anterior descending artery lesions is reflecting the wider use of FFR in this vessel as a larger retrospective trial has already observed [23]. An inherent referral bias cannot be excluded as only patients with lesions deemed to be significant in cCTA were further sent for clinical invasive clarification. $\mathrm{FFR}_{\mathrm{CT}}$ measurements for both kernels were done by the same operator, so bias cannot be excluded totally, although the operator was strictly blinded for the invasive FFR value. Moreover, we analyzed the influence of the reconstruction kernel on $\mathrm{FFR}_{\mathrm{CT}}$ values within the same data set. The potential influence of other acquisition parameters on CT-derived FFR including tube current and output, and collimation and contrast opacification of the coronary arteries, was not analyzed in this cohort. Furthermore, this is a single-vendor study, and further research is needed to generalize the results to other software simulating FFR $_{\mathrm{CT}}$.

In conclusion, we could demonstrate that image reconstruction parameters have a significant impact on the diagnostic performance of $\mathrm{FFR}_{\mathrm{CT}}$. A sharper reconstruction kernel provides a higher correlation with invasive FFR, potentially changing clinical decision-making.

Funding Open Access funding enabled and organized by Projekt DEAL.

\section{Declarations}

Guarantor The scientific guarantor of this publication is Fabian Ammon.

Conflict of interest Fabian Ammon received speaker honoraria from Edwards Lifescience. Mohamed Marwan received speaker honoraria from Siemens Healthineers and Edwards Lifescience. The other authors have nothing to disclose.

Statistics and biometry No complex statistical methods were necessary for this paper.

Informed consent Written informed consent was not required for this study because of the retrospective design of only clinically available data.

Ethical approval Institutional Review Board approval was not required because of the retrospective design of only clinically available data

\section{Methodology}

- retrospective

- diagnostic study

- performed at one institution

Open Access This article is licensed under a Creative Commons Attribution 4.0 International License, which permits use, sharing, adaptation, distribution and reproduction in any medium or format, as long as you give appropriate credit to the original author(s) and the source, provide a link to the Creative Commons licence, and indicate if changes were made. The images or other third party material in this article are included in the article's Creative Commons licence, unless indicated otherwise in a credit line to the material. If material is not included in the article's Creative Commons licence and your intended use is not permitted by statutory regulation or exceeds the permitted use, you will need to obtain permission directly from the copyright holder. To view a copy of this licence, visit http://creativecommons.org/licenses/by/4.0/.

\section{References}

1. Bech GJ, De Bruyne B, Pijls NH et al (2001) Fractional flow reserve to determine the appropriateness of angioplasty in moderate coronary stenosis: a randomized trial. Circulation 103:2928-2934

2. Zimmermann FM, Ferrara A, Johnson NP et al (2015) Deferral vs. performance of percutaneous coronary intervention of functionally non-significant coronary stenosis: 15 -year follow-up of the DEFER trial. Eur Heart J 36:3182-3188 
3. Tonino PA, De Bruyne B, Pijls NH et al (2009) Fractional flow reserve versus angiography for guiding percutaneous coronary intervention. N Engl J Med 360:213-224

4. Pijls NH, Fearon WF, Tonino PA et al (2010) Fractional flow reserve versus angiography for guiding percutaneous coronary intervention in patients with multivessel coronary artery disease: 2-year follow-up of the FAME (Fractional Flow Reserve Versus Angiography for Multivessel Evaluation) study. J Am Coll Cardiol $56: 177-184$

5. Authors/Task Force members, Windecker S, Kolh P et al (2014) 2014 ESC/EACTS Guidelines on myocardial revascularization: the Task Force on Myocardial Revascularization of the European Society of Cardiology (ESC) and the European Association for Cardio-Thoracic Surgery (EACTS) developed with the special contribution of the European Association of Percutaneous Cardiovascular Interventions (EAPCI). Eur Heart J 35:2541-2619

6. Min JK, Taylor CA, Achenbach S et al (2015) Noninvasive fractional flow reserve derived from coronary CT angiography: clinical data and scientific principles. JACC Cardiovasc Imaging 8:1209-1222

7. Nakazato R, Park HB, Berman DS et al (2013) Noninvasive fractional flow reserve derived from computed tomography angiography for coronary lesions of intermediate stenosis severity: results from the DeFACTO study. Circ Cardiovasc Imaging 6:881-889

8. Norgaard BL, Leipsic J, Gaur S et al (2014) Diagnostic performance of noninvasive fractional flow reserve derived from coronary computed tomography angiography in suspected coronary artery disease: the NXT trial (Analysis of Coronary Blood Flow Using CT Angiography: Next Steps). J Am Coll Cardiol 63:1145-1155

9. Leipsic J, Yang TH, Thompson A et al (2014) CT angiography (CTA) and diagnostic performance of noninvasive fractional flow reserve: results from the Determination of Fractional Flow Reserve by Anatomic CTA (DeFACTO) study. AJR Am J Roentgenol 202:989-994

10. Hawass NE (1997) Comparing the sensitivities and specificities of two diagnostic procedures performed on the same group of patients. Br J Radiol 70:360-366

11. Baumann S, Wang R, Schoepf UJ et al (2015) Coronary CT angiography-derived fractional flow reserve correlated with invasive fractional flow reserve measurements-initial experience with a novel physician-driven algorithm. Eur Radiol 25:1201-1207

12. Renker M, Schoepf UJ, Wang R et al (2014) Comparison of diagnostic value of a novel noninvasive coronary computed tomography angiography method versus standard coronary angiography for assessing fractional flow reserve. Am J Cardiol 114:1303-1308
13. Rother J, Moshage M, Dey D et al (2018) Comparison of invasively measured FFR with FFR derived from coronary CT angiography for detection of lesion-specific ischemia: results from a PC-based prototype algorithm. J Cardiovasc Comput Tomogr 12:101-107

14. Pijls NH, De Bruyne B, Peels K et al (1996) Measurement of fractional flow reserve to assess the functional severity of coronaryartery stenoses. N Engl J Med 334:1703-1708

15. Tonino PA, Fearon WF, De Bruyne B et al (2010) Angiographic versus functional severity of coronary artery stenoses in the FAME study fractional flow reserve versus angiography in multivessel evaluation. J Am Coll Cardiol 55:2816-2821

16. Knuuti J, Wijns W, Saraste A et al (2020) 2019 ESC guidelines for the diagnosis and management of chronic coronary syndromes. Eur Heart J 41:407-477

17. Meijboom WB, Meijs MF, Schuijf JD et al (2008) Diagnostic accuracy of 64-slice computed tomography coronary angiography: a prospective, multicenter, multivendor study. J Am Coll Cardiol 52:2135-2144

18. Douglas PS, Hoffmann U, Patel MR et al (2015) Outcomes of anatomical versus functional testing for coronary artery disease. N Engl J Med 372:1291-1300

19. The SCOT-HEART investigators (2015) CT coronary angiography in patients with suspected angina due to coronary heart disease (SCOT-HEART): an open-label, parallel-group, multicentre trial. Lancet 385:2383-2391

20. SCOT-HEART Investigators, Newby DE, Adamson PD et al (2018) Coronary CT angiography and 5-year risk of myocardial infarction. N Engl J Med 379:924-933

21. Curzen NP, Nolan J, Zaman AG, Norgaard BL, Rajani R (2016) Does the routine availability of CT-derived FFR influence management of patients with stable chest pain compared to CT angiography alone?: the FFRCT RIPCORD Study. JACC Cardiovasc Imaging 9:1188-1194

22. Douglas PS, De Bruyne B, Pontone G et al (2016) 1-year outcomes of FFRCT-guided care in patients with suspected coronary disease: the PLATFORM Study. J Am Coll Cardiol 68:435-445

23. Biasco L, Pedersen F, Lonborg J et al (2015) Angiographic characteristics of intermediate stenosis of the left anterior descending artery for determination of lesion significance as identified by fractional flow reserve. Am J Cardiol 115:1475-1480

Publisher's note Springer Nature remains neutral with regard to jurisdictional claims in published maps and institutional affiliations. 